\title{
EXPERIENCE WITH THE AVOIDANCE OF ALLERGIC REACTIONS TO PYELOGRAPHIC MEDIA BY THE USE OF ANTIHISTAMINE DRUGS
}

\author{
Reed M. Nesbit \\ Unicersily of Michigan, Ann Arbor, Mich.
}

All contrast media used for excretory pyelograms evoke undesirable side reactions in some patients. Many are mild and transient reactions, such as flushing, a feeling of warmth, metallic taste, arm pain, nausea, and vomiting. The more serious side reactions include urticaria, asthma, and shock; on rare occasions these result in death. Recently new compounds have been developed that consistently yield pyelograms of excellent quality and also appear to evoke fewer reactions than compounds earlier available. ${ }^{1,2}$

I recently collected data on the occurrence of severe reactions from members of the American Association of Genito-Urinary Surgeons, Boston, Mass. The purpose of the survey was to determine, from the experience of these urologists, the value of tests for predicting reactions, as well as to discover the prevalent attitude on the value of antihistamine drugs in their prevention. A report of this survey will be published in the proceedings of the association. TABLE 1 shows the data obtained from the survey.

The majority of those reporting expressed some degree of enthusiasm regarding the lack of reactions that have occurred with the new agents, yet there is about a 1 -in-500 over-all incidence of severe reactions, and none of the compounds is free of this risk.

The death occurred in a case where $1 \mathrm{cc}$. of Hypaque was introduced intravenously as a test and no reaction occurred. The final injection was given 4.5 min. later and, within $5 \mathrm{~min}$. the patient had a circulatory collapse. Oxygen, intravenous fluids, and Neo-Synephrine were administered and, finally, cardiac massage by the open method was done. An authority ${ }^{3}$ in the field of allergy research has informed me that this death probably was not due to an antigenantibody or histamine release mechanism for the reason that no reaction of an allergic nature was evoked by the preliminary test dose. It was postulated that a serotonin or a direct cellular histamine or some other chemical release might have been the basis for the reaction, and that it probably would not have been prevented by antihistamine injection. Another possibility is that the newer urographic media may have some direct depressing effect upon the cardiovascular system similar to the effects of Diodrast. Various investigators ${ }^{4,5}$ have demonstrated that Diodrast is a potent vasodilating agent that produces a fall in blood pressure and changes in the arterial pulse contour. The Diodrast reaction, particularly in humans, is suggestive of a parasympathetic discharge such as would occur, for example, after the administration of acetyl-betamethylcholine. The effects are a drop in blood pressure, bradycardia, nausea, flushing, cough, dyspnea, and sweating. Gordon et al. ${ }^{6}(1950)$, who studied the effects of Diodrast in animals, were unable to prevent their occurrence with atropine, vagotomy, or tetraethylammonium and concluded that the peripheral 
Nesbit: Avoidance of Allergic Reactions

TABLE 1

Data Obtained From Survey

\begin{tabular}{|c|c|c|c|c|c|}
\hline \multirow{2}{*}{$\begin{array}{l}\text { No. } \\
\text { jnvestigators } \\
\text { reporting }\end{array}$} & \multirow{2}{*}{ Medium } & \multirow{2}{*}{ No. of injections } & \multicolumn{2}{|c|}{ Severe reactions } & \multirow{2}{*}{ Deaths } \\
\hline & & & Allergic & Shock & \\
\hline 41 & Hypaque & 111,000 & 150 & 117 & 1 \\
\hline 12 & Miokon & 20,000 & 13 & 15 & 0 \\
\hline 10 & Renografin & 13,000 & 2 & 4 & 0 \\
\hline 63 & & 144,000 & 165 & 136 & 1 \\
\hline
\end{tabular}

effects are probably exerted directly upon the smooth muscle of the vessel walls. These investigators were unable to prevent the "Diodrast reaction" in animals with Pitressin, sympathomimetic drugs, or epinephrine. In fact, they found that the secondary vasodepressor action of epinephrine potentiates that of Diodrast and suggested that epinephrine might be harmful rather than beneficial in treating this type of reaction clinically. Pendergrass ${ }^{7,8}$ estimates the incidence of fatal reactions as 8.6 per million urograms.

In our survey forty-one reported the use of some test procedure for predicting sensitivity to the compounds. Many employ intradermal or conjunctival testing, yet only two believe that these tests have any value. It appears that these tests are performed not so much for patient protection as for legal protection of the doctors. Pendergrass reported that sensitivity tests were negative in about two thirds of the cases that died. It should be pointed out that most of the tests in question are tests of allergic sensitivity and that they are probably reliable indices of this type of reaction. However, the shock reaction, which often is associated with fatality, is probably not an antigen-antibody response and so would not be predictable by the usual allergy tests.

Many of the urologists canvassed in our survey inject $1 \mathrm{cc}$. or less of the contrast medium as a test for reactions, and the majority believe that this is a reliable indication. Yet 14 men point out that this is completely unreliable. In this connection 29 of the 61 fatal reactions reported recently by Pendergrass et al. $(1958)^{8}$ occurred in patients to whom intravenous test doses of $1 \mathrm{cc}$. of contrast medium were given and no reactions were observed. Pendergrass also reports that 3 of the fatal reactions occurred in persons administered less than $2 \mathrm{cc}$. of the contrast medium.

These observations fit into the concept that the shock reaction may be due to a drug idiosyncrasy or intolerance and not an immunological mechanism. Idiosyncrasy has been defined ${ }^{9}$ as denoting a qualitative alteration in the response of the individual to the pharmacological or toxic actions of a drug. Some patients experience the usual therapeutic or toxic effects of a drug at dosage levels either much lower or much higher than that usually required to produce these effects. If shock reactions are indeed due to idiosyncrasy, then a 1-cc. dose might not evoke a severe reaction in one patient, while the same amount might be fatal in another.

Results obtained by our group with antihistamine drugs for preventing reactions have been varied. Nearly every person who reported the use of anti- 
histamines expressed a belief that they are distinctly valuable. Those who administer the drugs orally appear to be the least enthusiastic as a group, yet some of these have a high regard for the method. It would appear that oral premedication has been most effective when employed in fairly large doses given at least 1 hour before the injection of the contrast medium. One member of our group reported that he gives $100 \mathrm{mg}$. of pyrobenzamine orally to all patients who have a history of allergy, and that he has observed excellent and uniform protection from reactions in these patients.

While visiting England in July, 1957, I made the rounds at The London Hospital, where it was revealed that antihistamine premedication is employed in all patients who have a history of allergy. Such patients are given $25 \mathrm{mg}$. of Phenergan by mouth the night before and another $25 \mathrm{mg}$. the morning of the injection; an intramuscular injection of $25 \mathrm{mg}$. is then given $20 \mathrm{~min}$. prior to the injection of the urographic medium (Hypaque). Intramuscular injection of Phenergan is preferred to intravenous as it is thought to afford a longer period of protection. When this medication has been employed in patients who give a history of allergy there have been no reactions.

In our survey, the urologists who have employed antihistamine drugs intravenously have had better results in the aggregate than those who have given them orally. There are 2 methods of administering the compounds intravenously; most popular is that of mixing the antihistamine with the contrast medium for simultaneous injection. In the other method the antihistamine agent is administered intravenously 3 to $5 \mathrm{~min}$. before giving the iodine compound. Investigators in the field of allergy state that the latter procedure offers greater protection against histamine release than does simultaneous injection; clinical experience would appear to bear out that assertion. Our members who have given antihistamine drugs simultaneously with the injections of the contrast media have found a lessened incidence of reactions; however, a few have observed that reactions occurred often enough to raise a doubt as to the value of the method.

Among those who have administered the drugs intravenously as a premedication there has been unanimous agreement concerning the value of antihistamine protection. The largest series reported was that of my colleagues, ${ }^{10}$ who employed $50 \mathrm{mg}$. Benadryl in $1 \mathrm{cc}$. of water intravenously as a premedication in more than 3000 cases where 70 per cent Urokon was used. These patients, observed at the Wayne County General Hospital, Eloise, Mich., showed only 2 allergic reactions and no shock reactions. There was 1 mild urticaria and 1 severe urticaria with bronchial spasm that was relieved by adrenalin. One can only speculate upon the nature of the reaction that might have occurred in this patient had not Benadryl been competing for histamine that was released by the iodine compound. In this connection, some investigators ${ }^{11}$ believe that anaphylactic or allergic reactions cannot be entirely avoided by the administration of antihistamine drugs since it has been shown experimentally that maximum effective doses of these drugs do not completely eliminate the action of injected histamine.

One of our members reported that he gives $10 \mathrm{mg}$. of Chlor-Trimeton intravenously as a premedication in all cases with a history of allergy and has observed 
TABLE 2

Results with Premedication Compared with Contrors

\begin{tabular}{|c|c|c|c|c|c|}
\hline \multirow{3}{*}{ Premedication } & \multirow{3}{*}{ No. of cases } & \multirow{3}{*}{ Medium } & \multicolumn{3}{|c|}{ Percentages } \\
\hline & & & $\begin{array}{l}\text { Allergic } \\
\text { reactions }\end{array}$ & Sid & iects \\
\hline & & & $\begin{array}{c}\text { Urticaria } \\
\text { and dyspnea }\end{array}$ & Nausea & Vomiting \\
\hline Controls, no antihistamine & $\begin{array}{l}364 \\
350\end{array}$ & $\begin{array}{l}\text { Hypaque } \\
\text { Miokon }\end{array}$ & $\begin{array}{l}3.1 \\
2.0\end{array}$ & $\begin{array}{l}13 \\
22\end{array}$ & $\begin{array}{l}2.2 \\
3.7\end{array}$ \\
\hline $12 \mathrm{mg}$. Phenergan, I.V. & $\begin{array}{l}543 \\
417\end{array}$ & $\begin{array}{l}\text { Hypaque } \\
\text { Miokon }\end{array}$ & $\begin{array}{l}0.5 \\
0.5\end{array}$ & $\begin{array}{l}2.8 \\
8.9\end{array}$ & $\begin{array}{l}0.0 \\
0.5\end{array}$ \\
\hline
\end{tabular}

only 1 reaction (mild) in this group of patients. Another has given the same premedication in 600 cases with histories of allergy, and only 1 patient had any reaction-a single hive.

Some of the lesser reactions such as nausea and vomiting and arm pain appear to be unaffected by Benadryl or Chlor-Trimeton. Lapides ${ }^{12}$ compared the incidence of these reactions in the 70 per cent Urokon cases and found that nausea and vomiting occurred just as often in the premedicated patients as among those who had Urokon alone.

At the University Hospital, Ann Arbor, Mich., we are observing a series of cases that are premedicated with Phenergan, an antihistamine drug which has a profound antiemetic property. Twelve milligrams of Phenergan is administered intravenously about $5 \mathrm{~min}$. before the pyelographic medium is injected. Nine hundred and sixty patients have been treated in this manner and there appears to have been a significant decrease in the occurrence of nausea and vomiting. TABLF 2 indicates the observations made on a control series, as well as the cases premedicated with Phenergan.

Together with the marked decrease in occurrence of nausea and vomiting there has been observed a significantly decreased incidence of allergic reactions. However, our Phenergan series at the University Hospital has displayed a distinctly greater occurrence of reactions than has the Benadryl series at Wayne County General Hospital where only 2 very minor allergic reactions have occurred in more than 3000 injections. The low incidence of reactions in the latter series is all the more impressive considering the fact that 70 per cent Urokon has been used in all the cases. Qualitative differences in the hospital populations of the 2 institutions may account in part for some of these differences. In both institutions the contrast media are administered by members of the resident staff who observe the patients carefully and record data on every case. We now plan to use Benadryl at the University Hospital together with $\mathrm{Hy}$ paque and Miokon on alternate cases to determine whether the same protection from all types of reaction will prevail among the University Hospital population with these less toxic agents. The results of this study will be published.

Most of the reactions that have been observed in the University Hospital Phenergan series have been of a very mild nature and have given no cause for 
alarm. However, there have been 4 cases of a more severe nature that should be described in detail since some of the particulars may be of value in a longterm study elucidating the nature of severe or fatal reactions. The statistically minded will find interesting the fact that the reactions in question occurred in the last 100 cases of our series, all within a 3 -week period.

The first patient of the 4 showing severe reactions is a 17 -year-old female who was being studied because of recurrent urinary-tract infection. Her general physical condition was good and her renal function, determined by P.S.P. and creatinine tests, was normal. There were a few patch areas of atopic eczema of the skin, and blood counts were normal.

After the intravenous injection of $0.5 \mathrm{cc}$. $(12.5 \mathrm{mg}$.) of Phenergan she received $28 \mathrm{cc}$. of 50 per cent Hypaque. A very slow injection of the contrast medium was employed because of a history of atopic eczema since early childhood, multiple food intolerances, and various allergies to plant pollens and dust. She had never had a complete allergic investigation with skin tests.

Shortly after the injection was finished she complained of nausea and, $1 \mathrm{~min}$. later, vomited. Two minutes later she had a sensation of swelling of the lips and throat and difficulty in breathing. No swelling could be observed by the attending physician and auscultation of the chest revealed normal breath sounds, good air exchange, and normal thoracic excursions. The pulse was 92 and blood pressure 98/58. Within an additional $2 \mathrm{~min}$. she began to writhe in pain and complained of a severe burning sensation extending from the throat to the symphysis pubis. The pain rapidly increased in severity and concentrated in the suprapubic area. Catheterization of the bladder was done-60 cc. of fluid was obtained--but the pain was not relieved. At this point the femoral pulsations were found to be good, the bowel sounds were normal, and the blood pressure was $128 / 68$. Ten minutes after completion of the contrast medium injection the abdominal pain began to subside but the patient developed circumoral pallor, conjunctival injection, a red blush over the face and neck, and urticarial lesions of the chest, arms, and legs. Benadryl, $50 \mathrm{mg}$. in $5.0 \mathrm{cc}$. of water, was administered intravenously, and within $2 \mathrm{~min}$. the cutaneous lesions had almost completely disappeared. At $20 \mathrm{~min}$. after injection of the contrast medium the patient was resting quietly but complained of a chill, weakness, and general malaise. At $80 \mathrm{~min}$. the only residual symptoms were a cold sensation and drowsiness. This somnolence was thought to be due to the soporific action of the antihistamine agents.

This patient had been examined by the Allergy Section of the University Hospital since the reaction noted above, and skin tests have demonstrated a sensitivity to the iodine compound that was used.

The next patient is a 30 -year-old woman who was being investigated for ascites. The past history included no known allergies, although she was reported to have been treated in 1954 for a "nervous dermatitis" with eczematoid features, which began with urticaria and edema of the face and eyelids and was treated with cortisone. Shortly after injection of $12.5 \mathrm{mg}$. of Phenergan she coughed but displayed no other reaction. After the injection of 30 cc. of 50 per cent Miokon she complained of nausea and experienced some retching but no emesis. The development of a burning mid-chest pain was 
not accompanied by abnormal findings on examination of the chest; at that time, the blood pressure was $100 / 70$ and the pulse 88 . A few minutes later there was a diffuse red flush over her body and she had a hot sensation over the body, the face, and neck. There then developed marked pruritis but no urticarial lesions or hives. Benadryl, $50 \mathrm{mg}$. was given intravenously, and the symptoms abated. About $15 \mathrm{~min}$. after the injection of the contrast medium additional symptoms of dizziness, general malaise, and tightness of the skin of the face were mentioned. Shortly thereafter puffiness of the upper face and eyelids (angioneurotic edema) was in evidence. An additional $50 \mathrm{mg}$. of Benadryl was given intravenously with no significant effect. One hour after injection of the contrast medium there occurred a mild intermittent shaking chill and numbness of the face. The patient's vital signs remained stable and, after 12 hours of sleep, she had no residual symptoms. Later, this patient was investigated by our Allergy Service and the results of tests of sensitivity to the contrast medium were negative.

The third patient was a 27 -year-old woman who was investigated because of a chronic urinary-tract infection. She had had a nephrectomy 5 years before for a congenital anomaly of the kidney. There was a history of intolerance to sulfa drugs, which produced nausea but no other reactions. No allergies were reported by the patient and her normal blood pressure was 102/76.

This woman had premedication with $12.5 \mathrm{mg}$. of Phenergan intravenously. She received $30 \mathrm{cc}$. of 50 per cent Miokon and shortly thereafter became diaphoretic and pale and complained of a cold sensation. Her pulse became weak and rapid, although the blood pressure was $130 / 70$. She was given $0.3 \mathrm{cc}$. of adrenalin 1:1000 solution and her symptoms gradually abated. About 30 min. after the reaction began her blood pressure was $98 / 60$ and she complained of a sensation of coldness; no other symptoms occurred, and she felt well 3 hours later.

The fourth patient was an 8-year-old boy who had chronic glomerulotubular nephritis. His premedication consisted of $0.25 \mathrm{cc} .(6 \mathrm{mg}$.) of Phenergan, and he was given $15 \mathrm{cc}$. of 50 per cent Miokon. Following this he exhibited a red flush of the head and neck and, 2 min. later, developed a severe occipital headache. The blood pressure at this time was $142 / 82$. The boy was sat upright and was given spirits of ammonia to inhale; the headache subsided within a few minutes and the blood pressure dropped to $112 / 68$. He then became sleepy and slept most of the day. The following morning he appeared to have no symptoms. There was no history of allergy in this case.

The first of these cases was doubtless an allergic reaction to the pyelographic medium. It abated promptly following intravenous Benadryl. The second case appears not to have been allergic, for the patient was not benefited by Benadryl administration and was not found (by skin tests) to be sensitive to Miokon. The other two reactions were certainly not allergic in nature; one probably was merely vertigo while the last, with headache and slight elevation of the blood pressure, remains unexplained.

The protective value of antihistamine drugs in patients who have had previous reactions has been demonstrated in but few reported cases. Olsson $(1951)^{13}$ reported on "a number of cases" who had experienced reactions to the 
same medium; these patients, when protected by $20 \mathrm{mg}$. of Benadryl, suffered no discomfort. This investigator expressed the opinion that $20 \mathrm{mg}$. of Benadryl administered intravenously is adequate protection against any amount or repeated injections of the medium. My information, obtained from experts in the field of allergic research, is that Benadryl should be administered intravenously in the amount of $50 \mathrm{mg}$. if it is to afford maximum effective protection when used as a premedication.

A significant case in the category of protection was reported to me by Melvin Figley, of the University of Washington Medical School, Seattle, Wash. While at Ann Arbor, Figley supervised all the arteriography performed at the University Hospital. He observed a severe shock reaction following arteriography with 70 per cent Urokon in a young man examined for a vascular anomaly. A repeat arteriogram became necessary at a later date, and $50 \mathrm{mg}$. of Benadryl was administered intravenously a few minutes before the Urokon injection; no reaction occurred.

Another similar experience was observed in a student at the University of Michigan who had a severe allergic reaction following pyelography with Hypaque. Later, another pyelographic study was carried out with Hypaque but, this time, intravenous premedication with $50 \mathrm{mg}$. of Benadryl was used and the patient had no reaction.

A third case in my experience was a dramatic error, fortunately not fatal. The patient had her ureter injured during a laparotomy for pelvic neoplasm; this was successfully repaired. During check-up pyelograms she went into severe shock following Miokon injection and apparently was saved by prompt oxygen administration by an anesthetist who chanced to be present at the time. Later, the patient was seen in the Urology Clinic for check-up and was again given Miokon by a resident surgeon who was not informed of the reaction that had occurred 3 months before. Again she went immediately into a severe state of shock and again was apparently saved by the fortuitous presence of an anesthetist who administered artificial respiration and oxygen. A few months later she was re-examined. This time $50 \mathrm{mg}$. of Benadryl was given as premedication; Hypaque was employed as the medium and there was no reaction. Subsequently the patient was examined on the Allergy Service and was found to react severely to skin tests with both. Miokon and Hypaque.

A disadvantage of antihistamine administration is the soporific effect that sometimes occurs. Occasionally patients become quite sleepy when these drugs are administered by any route. This effect has no great disadvantage in the hospitalized patient but can be disturbing or even potentially dangerous to the ambulatory patient, particularly one who has driven an automobile to the $\mathrm{X}$-ray laboratory. When ambulatory patients are given these drugs they should be observed for a short period of time and, if sleepy, should be given amphetamine to counteract the somnolent effects. Thereafter they should not drive a motor car for several hours.

\section{Discussion}

It appears evident that there are at least two distinct types of severe reactions that are evoked by intravenous administration of urographic media. 
One is an antigen-antibody reaction that can be severe in nature and probably fatal in certain cases. This allergic reaction can probably always be predicted by standard allergy tests for specific sensitivity; it can generally be prevented by antihistamine drugs and can be ameliorated or terminated by antihistamine therapy. ${ }^{14-16}$

The second type of reaction, the so-called shock reaction, is not allergic in nature. This reaction, which also may be fatal, probably is due to drug idiosyncrasy; it is not predictable by known methods of preinjection testing and does not appear to be responsive to antihistamine or other chemical methods of treatment. Whether its occurrence can be prevented by premedication is not known.

The study reported in this article has revealed that the urologists who have employed antihistamine drugs to avoid or diminish reactions with urographic media are convinced of the value of these drugs. The reports suggest that oral administration affords the least protection and that premedication by intravenous injection is better than the simultaneous administration of the antihistamine with the iodine compound.

My studies with Phenergan as a premedication in nearly 1000 cases have demonstrated that this compound will reduce substantially the incidence of allergic reactions to the urographic agents that have been tested. Also, the antiemetic properties of Phenergan appear to have reduced the incidence of nausea and vomiting. However, a few reactions of a severe type were observed in the Phenergan series. In comparison, the Benadryl series by colleagues in a nearby hospital suggests the possibility that this antihistamine may possess protective properties that are of a superior order for, among 3025 cases, there were only 2 allergic reactions and no shocklike reactions. It is possible that this may be related to the atropine effect of Benadryl, which is greater than the atropine effect of Phenergan. It is also possible that the dosage of Benadryl bears an important relation to its usefulness in this connection. Hart et al. $(1958)^{17}$ recently reported a series of more than 4000 cases in which a variety of urographic media was used and in which Chlor-Trimeton as well as Benadryl was used to prevent reactions. These investigators found that the antihistamine drugs were beneficial in reducing incidence and severity of reactions, but did not eliminate them entirely. One of their patients, who had Benadryl simultaneously with Urokon, suffered a shock reaction. The patients were given only $10 \mathrm{mg}$. of Benadryl, which appears to be a very small dose.

My observations suggest that Benadryl in proper dosage and administered intravenously may afford maximum protection against not only allergic but also the so-called shock reactions that occur with urographic media. Whether all fatal reactions or, indeed, all shocklike reactions could be eliminated by this means may be determined only by the observation of an enormous number of cases: it should be recalled that Pendergrass estimated the incidence of deaths at 8.6 per million urograms. It is interesting to observe that none of the fatal cases listed by Pendergrass was reported to have had antihistamine premedication.

The evidence in favor of utilizing antihistamine premedication appears to suggest that serious reactions and deaths from reactions to urographic media 
can be reduced, if not entirely eliminated. Only the widespread use of these preventive techniques will reveal their ultimate worth.

Roentgenologists and urologists in the United States could determine the ultimate value of antihistamine protection within the course of one or two years if a large number of them would administer these drugs as routine and report the occurrence of any adverse reactions or deaths to their respective societies. Only by obtaining the record of an enormous number of cases can this important matter be settled. This objective is worthy of our professional effort.

\section{References}

1. Borne, A. W. \& W. W. Christeson. 1953. Clinical evaluation of a concentrated iodine preparation. Radiology. 60: 401 .

2. UTz, C. \& G. J. Tirompson. 1958. Evaluation of contrast media for excretory urography. Proc. Staff Meetings Mayo Clin. 33(4): 75.

3. SHeldon, J. M. Personal communication.

4. Bersack, S. W. \& T. E. Whitaker, JR. 1953. Effect of diphenhydramine (Benadryl) on side-reactions in intravenous urography. A.M.A. Arch. Internal. Med. 91: 618.

5. Edwards, E. A. \& F. Bigtria. 1934. Comparison of Skiodan and Diodrast as urographic media, with special reference to their effect on blood pressure. New Engl. J. Med. 211: 589 .

6. Gordon, A. J., S. A. Bratims, S. Megtbow \& M. L. Sussman. 1950. An experimental study of the cardiovascular effects of Diodrast. Am. J. Roentgenol. 64(5): 819 .

7. Pendergrass, E. P., P. J. Hodes, R. L. Tondreat, C. C. Powell, \& E. D. Burdick, 1955. Further consideration of deaths and unfavorable sequelae following the administration of contrast media in urography in the United States. Am. J. Roentgenol. 74: 262.

8. Pendergrass, H. P., R. L. Tondreau, F. P. Pfendergrass, D. J. Richie, E. A. Hildretit, \& S. I. Askovitz. 1958. Reactions associated with intravenous urography: historical and statistical review. Radiology. 71(1): 1 .

9. Sheidon, J. \& L. Matthews. 1953. Manual of Clinical Allergy. Saunders. Philadelphia, Pa.

10. LAPIDES, J. \& R. E. BOYD. 1956. The effect of Benadryl in allaying allergic manifestations of 70 per cent Urokon. J. Urol. $75(6): 1016$.

11. Wells, J. A., H. C. Morris \& C. A. Dragstejy. 1946. Modification of anaphylaxis by Benadryl. Proc. Soc, Exptl. Biol. Med. 61: 101.

12. LAPIDES, J. Personal communication.

13. Olsson, $O$. 1951. Antihistaminic drugs for inhibiting untoward reactions to injections" of contrast medium. Acta Radiol. 35: 65 .

14. Getzoff, P. L. 1951. Use of antihistamine drug prophylaxis against I iodrast reac tions. J. Urol. 65: 1139.

15. Simon, S. W., H. I. Berman \& S. A. Rosenblum. 1954. Prevention of reactions in intravenous urography. J. Allergy, 25: 395 .

16. WINTER, C. C. 1955. The value of Chlor-Trimeton in the prevention of immediate reactions to 70 per cent Lrokon. J. Urol. 74: 416.

17. HART, L., M. D. Sachs \& H. GRabstalDT. 1958. Comparative study of urographic contrast media. A.M.A. Arch. Surg. 77(1): 75 . 